\title{
Correlation analysis of monocyte subsets and insulin resistance considering fetuin- $A$ involvement in patients with type 2 diabetes
}

CrossMark

\author{
Saori Maruo ${ }^{1}$, Katsuhito Mori2 ${ }^{2 *}$ Koka Motoyama' ${ }^{1}$ Miyuki Nakamura', Reina Kawarabayashi', \\ Yoshinori Kakutani ${ }^{1}$, Yuko Yamazaki ${ }^{1}$, Tomoaki Morioka ${ }^{1}$, Tetsuo Shoji ${ }^{3}$, Masaaki Inaba ${ }^{1,2}$ and Masanori Emoto ${ }^{1}$
}

\begin{abstract}
Background: Fetuin-A is a multifunctional circulating glycoprotein that can induce insulin resistance. Lately, adipose tissue has gained prominence as an effector site of fetuin-A. Although fetuin-A —induced proinflammatory polarization and migration of macrophages plays a crucial role, it remains obscure whether monocyte subsets in circulation could simulate characteristics of macrophages in adipose tissues. This study aims to investigate the correlation between monocyte subsets with fetuin-A and its relevant insulin resistance.

Results: We evaluated serum fetuin-A levels in 107 patients with type 2 diabetes (T2D). Using flow cytometry, we classified monocyte subsets into three subtypes: (a) classical, CD14 ${ }^{++}$CD $16^{-}$; (b) intermediate, CD $14^{++}{\text {CD } 16^{+}}$, the most proinflammatory one; (c) and nonclassical, $\mathrm{CD} 14^{+} \mathrm{CD} 16^{++}$. We assessed the insulin resistance by the homeostasis model assessment for insulin resistance (HOMA-IR) in 68 patients without insulin injections. We observed no correlation between fetuin-A levels and classical $(\rho=-0.005 ; P=0.959)$, intermediate $(\rho=0.022 ; P=0.826)$, and nonclassical monocyte counts $(\rho=0.063 ; P=0.516)$, respectively. In addition, no significant correlation was found between $\log (\mathrm{HOMA}-\mathrm{IR})$ and classical $(\rho=0.052 ; P=0.688)$, intermediate $(\rho=0.054 ; P=0.676)$, and nonclassical monocyte counts $(\rho=0.012 ; P=0.353)$, respectively. However, serum fetuin-A levels showed positive correlation with $\log (\mathrm{HOMA}-\mathrm{IR})(\rho=0.340 ; P=0.007)$. Multiple regression analyses revealed a significant relationship between fetuin-A and $\log (H O M A-I R)(\beta=0.313 ; P=0.016)$, but not with monocyte subsets.
\end{abstract}

Conclusions: Monocyte subsets in circulation, including proinflammatory intermediate monocytes, were not associated with fetuin-A and insulin resistance.

Keywords: Fetuin-A, Insulin resistance, CD14, CD16, Type 2 diabetes, Monocyte subsets

\section{Background}

Fetuin-A ( $\alpha 2$-Heremans Schmid glycoprotein: AHSG) is an abundant circulating glycoprotein that is primarily synthesized in the liver and plays several functions in human physiology and pathology [1]. Among these, insulin resistance induction is well recognized [2, 3]. Recently, adipose tissue, which is the primary inflammation site where infiltrated macrophages interact with adipocytes, has gained prominence as an effector site of

\footnotetext{
${ }^{*}$ Correspondence: ktmori@med.osaka-cu.ac.jp

2 Department of Nephrology, Osaka City University Graduate School of Medicine, 1-4-3, Asahi-machi, Abeno-ku, Osaka 545-8585, Japan Full list of author information is available at the end of the article
}

fetuin-A. Pal et al. demonstrated that fetuin-A acts as an essential adaptor protein when free fatty acids (FFA) bind to Toll-like receptor-4 (TLR4) in both adipocytes and macrophages, signifying that FFA-fetuin-A-TLR4 ternary complex with intracellular activation of NF- $\mathrm{kB}$ can provoke systemic insulin resistance by enhancing proinflammatory cytokine secretion [4]. Fetuin-A has been reported to act as a chemoattractant for macrophage migration into adipose tissue and, subsequently, polarize anti-inflammatory M2 to proinflammatory M1 macrophages [5]; this suggests that the pivotal role of fetuin-A in inflamed adipose tissue is a collaboration with macrophages. 
Monocytes, originally derived from the bone marrow, circulate in the bloodstream and infiltrate into tissues in response to environmental changes, resulting in differentiated macrophages [6]. Hence, the monocytemacrophage system is involved in various inflammatory diseases, including atherosclerosis [6]. Although monocytes are heterogeneous and diverse, various markers and different terms have confused monocyte classification. Accordingly, a consensus nomenclature for monocytes was proposed and approved, internationally, in 2010 to solve this problem [7]. At present, the following three distinct subsets of human monocytes are recognized according to the expression of CD14, a lipopolysaccharide co-receptor, and $\mathrm{CD} 16, \mathrm{FC} \gamma$-III receptor: (a) classical, CD14 ${ }^{++} \mathrm{CD}_{16}{ }^{-}$; (b) intermediate, $\mathrm{CD} 14^{++} \mathrm{CD} 16^{+}$; and (c) nonclassical, CD $14^{+} \mathrm{CD} 16^{++}$[7]. In comparison to others, $\mathrm{CD} 14^{++} \mathrm{CD} 16^{+}$monocytes show proinflammatory characteristics [8]. In fact, the elevated numbers of $\mathrm{CD} 14^{++} \mathrm{CD} 16^{+}$monocytes have been reported to be an independent predictor of cardiovascular events [9-11], suggesting a profound association between CD $14^{++}$CD $16^{+}$monocytes and atherosclerosis.

Although recent evidence supports that fetuin-A can elicit and amplify insulin resistance within adipose tissue, estimation of macrophage-driven adipose tissue inflammation without invasive techniques, such as biopsy, is challenging. We hypothesized that fetuin-A-induced polarization of macrophages from M2 to M1 in adipose tissue might be comparable to the proinflammatory shift of monocyte subsets by fetuin-A in the bloodstream. Hence, this study aims to elucidate whether circulating monocyte subsets, especially CD $14^{++} \mathrm{CD} 16^{+}$monocytes, are associated with serum fetuin-A levels and their related insulin resistance in patients with type 2 diabetes mellitus (T2D).

\section{Methods}

\section{Subjects}

In this study, we enrolled patients with T2D who admitted to our diabetes center at Osaka City University Hospital (Osaka, Japan) between October 2011 and April 2013. The diagnosis of diabetes was according to a history of diabetes or the American Diabetes Association criteria [12]. Notably, we excluded patients with serum creatinine levels $>1.5 \mathrm{mg} / \mathrm{dL}$ and other active medical diseases from this study. We calculated the estimated glomerular filtration rate (eGFR) per the guidelines of the Japanese Society of Nephrology [13]. This study was approved by the ethical review board of our institution (registration number 164), and we obtained informed consent from each patient.

\section{Biochemical analysis}

We determined serum fetuin-A using an enzymelinked immunosorbent assay kit (BioVender Laboratory
Medicine, Inc., Czech Republic) as previously described $[14,15]$.

\section{Homeostasis model assessment}

Based on Matthews et al. we calculated the Homeostasis model assessment for insulin resistance (HOMA-IR) from fasting plasma glucose (FPG) and immunoreactive insulin (FIRI), using the following formula [16]: HOMA$\mathrm{IR}=\mathrm{FIRI}$ in $\mu \mathrm{U} / \mathrm{mL} \times \mathrm{FPG}$ in $\mathrm{mmol} / \mathrm{L} / 22.5$.

\section{Peripheral blood monocyte cell isolation and flow cytometry analysis}

Peripheral blood samples were freshly collected from all patients after 8-h fasting period with ethylenediaminetetraacetic acid-containing (EDTA) tube. Erythrocytes were lysed using a lysing solution (lysing buffer; BD Pharm Lyse ${ }^{\mathrm{TM}}$; BD Biosciences, Japan) to segregate leukocytes. For the cytometric analysis, we used fluorescent-conjugated monoclonal antibodies against CD14 (PE-Cy7 Mouse Anti-Human CD14; BD Pharmingen ${ }^{\mathrm{TM}}$, BD Biosciences) and CD16 (APC-Cy7 Mouse AntiHuman CD16; BD Pharmingen ${ }^{\mathrm{TM}}$, BD Biosciences) for labeling monocyte subsets. We measured 100,000 labeled leukocytes for each patient by flow cytometry (BD FACSCanto ${ }^{\mathrm{TM}}$; Becton-Dickinson Bioscience, Japan). Monocytes were first gated by the forward scatter and sideward scatter dot plot. After that, according to the properties and the level CD14 and CD16 expression, we categorized monocytes into the following three subsets: (a) classical, $\mathrm{CD} 14^{++} \mathrm{CD} 16^{-}$; (b) intermediate, $\mathrm{CD}_{14}{ }^{++} \mathrm{CD} 16^{+}$; and (c) nonclassical, CD $14^{+} \mathrm{CD} 16^{++}$. We analyzed using FlowJo 7.5.5 (TreeStar Inc.) (Fig. 1a).

\section{Statistical analysis}

In this study, all values are presented as mean $\pm \mathrm{SD}$ or median (interquartile) as appropriate. Data were evaluated using JMP v.10 (SAS Institute Inc., Cary, NC). In addition, unpaired $t$ test and the Mann-Whitney $U$-test were used where appropriate. We used the Spearman's rank correlation test to evaluate the relationships among serum fetuin-A, monocyte subsets, and HOMA-IR. Log-transformation was performed to achieve a normal distribution of HOMA-IR, and multivariate regression analyses were performed to evaluate the correlation between log (HOMA-IR) and various parameters. We considered $P<0.05$ as statistically significant.

\section{Results}

Table 1 summarizes the clinical characteristics of the study population. We evaluated serum fetuin-A levels in 107 patients with type 2 diabetes (T2D). The mean serum levels of fetuin-A were $249 \pm 40 \mu \mathrm{g} / \mathrm{mL}$. The median monocyte count was $486(368-646)$ cells/ $\mu \mathrm{L}$, of which 
a

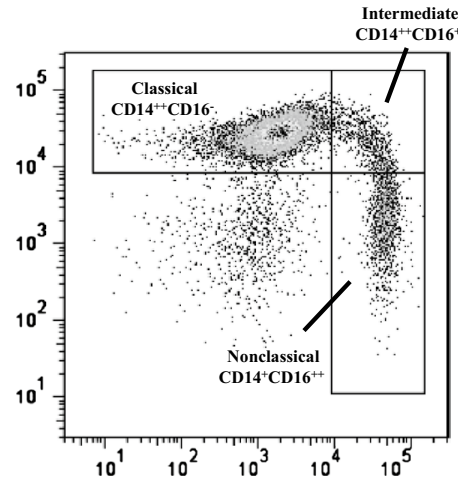

c

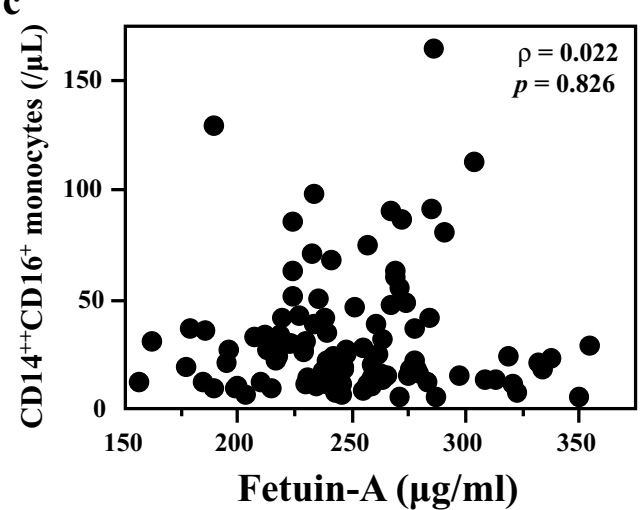

b

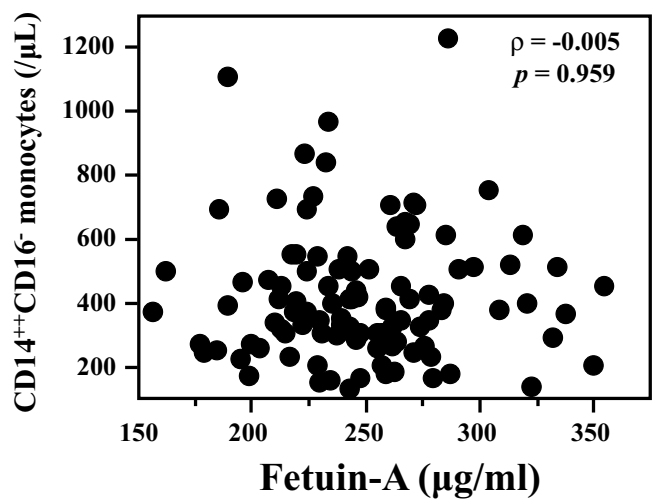

d

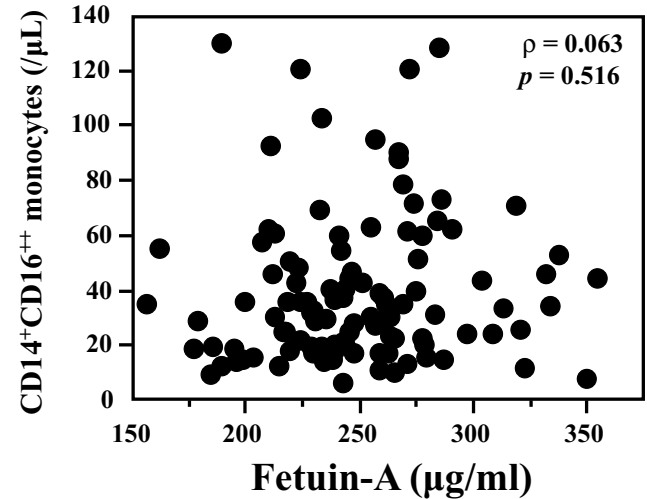

Fig. 1 The flow cytometric analysis of monocyte subsets (representative example; a) and the association of serum levels of fetuin-A with monocyte subsets (b-d)

379 (283-510) cells $/ \mu \mathrm{L}$ were classical $\mathrm{CD} 14^{++} \mathrm{CD} 16^{-}$ monocytes, 24 (15-39) cells $/ \mu \mathrm{L}$ were intermediate $\mathrm{CD} 14^{++} \mathrm{CD} 16^{+}$monocytes, and $33(20-51)$ cells $/ \mu \mathrm{L}$ were nonclassical $\mathrm{CD} 14^{+} \mathrm{CD} 16^{++}$monocytes. Of all patients, 39 were had undergone treatment with insulin injections. Regarding fetuin-A levels and monocyte subset counts, no difference was observed with or without insulin therapy.

Figure $1 \mathrm{~b}-\mathrm{d}$ shows the correlation between fetuin-A and monocyte subsets. We determined no association between fetuin-A levels and classical $\mathrm{CD} 14^{++} \mathrm{CD} 16^{-}$ monocyte counts $(\rho=-0.005 ; P=0.959)$, intermediate $\mathrm{CD} 14^{++} \mathrm{CD} 16^{+}$monocyte counts $(\rho=0.022$; $P=0.826)$, and nonclassical $\mathrm{CD} 14^{+} \mathrm{CD} 16^{++}$monocyte counts $(\rho=0.063 ; P=0.516)$, respectively. Although we divided the subjects into two subgroups (low fetuinA or high fetuin-A) and performed similar analyses, no significant association was found between fetuin-A and monocyte subsets in each subgroup. Since some medications might affect inflammatory state, serum fetuin-A levels, and distribution of monocyte subsets, we focused on insulin sensitizers (pioglitazone and metformin).
However, there was no correlation between fetuin-A and monocyte subsets in patients without insulin sensitizers. After that, we evaluated the association between fetuin-A and monocyte subsets with insulin resistance characterized by HOMA-IR in patients without insulin injection. We determined no significant association between $\log$ (HOMA-IR) and classical $\mathrm{CD} 14^{++} \mathrm{CD} 16^{-}$ monocyte counts $(\rho=0.052 ; P=0.688)$, intermediate $\mathrm{CD} 14^{++} \mathrm{CD} 16^{+}$monocyte counts $(\rho=0.054$; $P=0.676)$, and nonclassical $\mathrm{CD} 14^{+} \mathrm{CD} 16^{++}$monocyte counts $(\rho=0.012 ; P=0.353)$, respectively (Fig. $2 \mathrm{a}-\mathrm{c}$ ). In addition, no association was determined between log (HOMA-IR) and monocyte subsets in patients without insulin sensitizers. However, serum fetuin-A levels showed positive correlation with $\log$ (HOMA-IR) ( $\rho=0.340 ; P=0.007$; Fig. $2 \mathrm{~d}$ ). We performed multiple regression analyses to explore the impact of various clinical factors on insulin resistance. Although body mass index (BMI; $\beta=0.305 ; P=0.034$ ) and fetuin-A were significantly associated with $\log$ (HOMA-IR) $(\beta=0.313$; $P=0.016$ ), monocyte subsets were not (Table 2 ). 
Table 1 Clinical characteristics of the study population

\begin{tabular}{|c|c|c|c|}
\hline & Without insulin & With insulin & Total \\
\hline$n$ & 68 & 39 & 107 \\
\hline Age (years) & $57 \pm 14$ & $68 \pm 8^{*}$ & $61 \pm 13$ \\
\hline Duration (years) & $7(0-50)$ & $20(0-46)^{\#}$ & $10(0-50)$ \\
\hline $\mathrm{BMI}\left(\mathrm{kg} / \mathrm{m}^{2}\right)$ & $27 \pm 6$ & $25 \pm 4^{*}$ & $26 \pm 5$ \\
\hline $\mathrm{SBP}(\mathrm{mmHG})$ & $125 \pm 18$ & $136 \pm 21^{*}$ & $129 \pm 20$ \\
\hline $\mathrm{Cr}(\mathrm{mg} / \mathrm{dL})$ & $0.8 \pm 0.3$ & $0.8 \pm 0.2$ & $0.8 \pm 0.2$ \\
\hline $\mathrm{eGFR}\left(\mathrm{mL} / \mathrm{min} / 1.73 \mathrm{~m}^{2}\right)$ & $79 \pm 27$ & $65 \pm 18^{*}$ & $74 \pm 25$ \\
\hline $\mathrm{FPG}(\mathrm{mg} / \mathrm{dL})$ & $119(67-203)$ & $103(72-309)$ & $113(67-309)$ \\
\hline $\mathrm{HbA} 1 \mathrm{C}(\%)$ & $8.1 \pm 1.8$ & $8.7 \pm 1.4$ & $8.3 \pm 1.7$ \\
\hline $\mathrm{LDL}-\mathrm{C}(\mathrm{mg} / \mathrm{dL})$ & $107 \pm 38$ & $88 \pm 25^{*}$ & $100 \pm 35$ \\
\hline $\mathrm{TG}(\mathrm{mg} / \mathrm{dL})$ & $111(50-1919)$ & $101(46-303)$ & 109 (46-1919) \\
\hline $\mathrm{HDL}-\mathrm{C}(\mathrm{mg} / \mathrm{dL})$ & $40 \pm 11$ & $44 \pm 12$ & $41 \pm 11$ \\
\hline HOMA-IR & $2.2(0-8.3)$ & $\mathrm{N} / \mathrm{A}$ & $\mathrm{N} / \mathrm{A}$ \\
\hline Fetuin-A $(\mu \mathrm{g} / \mathrm{mL})$ & $249 \pm 37$ & $249 \pm 44$ & $249 \pm 40$ \\
\hline 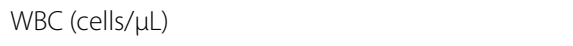 & $5800(4825-6775)$ & $5400(4500-7000)$ & $5700(4800-6800)$ \\
\hline Monocyte (cells/ $\mu \mathrm{L})$ & $472(372-648)$ & $492(350-616)$ & $486(368-646)$ \\
\hline 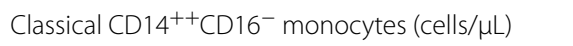 & $363(291-515)$ & $385(274-508)$ & $379(283-510)$ \\
\hline Intermediate CD14 ${ }^{++}$CD $16^{+}$monocytes (cells/ $\mu \mathrm{L}$ ) & $25(15-37)$ & $22(14-48)$ & $24(15-39)$ \\
\hline Nonclassical CD14+CD16 ${ }^{++}$monocytes (cells/ $\mu \mathrm{L}$ ) & $32(20-54)$ & $35(19-46)$ & $33(20-51)$ \\
\hline \multicolumn{4}{|l|}{ OADs: monotherapy } \\
\hline Biguanide (Met) & 9 & 2 & 11 \\
\hline Thiazolidine (Pio) & 0 & 0 & 0 \\
\hline Other OADs & 14 & 4 & 18 \\
\hline \multicolumn{4}{|l|}{ OADS: combination therapy } \\
\hline Met + other OADs & 14 & 5 & 19 \\
\hline Pio + other OADs & 2 & 0 & 2 \\
\hline Met + Pio \pm other OADs & 5 & 0 & 5 \\
\hline
\end{tabular}

Data are presented as mean \pm standard deviation, number, or median (interquartile range)

$S B P$ systolic blood pressure, $C r$ creatinine, $L D L-C$ low-density lipoprotein-cholesterol, $T G$ triglyceride, $H D L-C$ high-density lipoprotein-cholesterol, $W B C$ white blood cells, OADs Oral antidiabetic drugs, Met metformin, Pio pioglitazone

*, ${ }^{P} P<0.05$ versus group without insulin

\section{Discussion}

To the best of our knowledge, this is the first study to investigate the correlation of monocyte subsets with fetuin-A and insulin resistance in patients with T2D. This study determined no association of monocyte subsets, including $\mathrm{CD} 14^{++} \mathrm{CD} 16^{+}$monocytes, with serum fetuinA levels and HOMA-IR. Conversely, fetuin-A was found to be significantly contributing to insulin resistance.

Apparently, classical CD $14^{++} \mathrm{CD} 16^{-}$monocytes, which have the high antimicrobial capacity with phagocytosis of pathogens and production of antimicrobial proteins, leave the bone marrow and, subsequently, invade inflamed tissues and differentiated macrophages and/or dendritic cells. Alternatively, they differentiate into intermediate $\mathrm{CD} 14^{++} \mathrm{CD} 16^{+}$monocytes in the circulation. Perhaps, nonclassical CD $14^{+} \mathrm{CD} 16^{++}$monocytes are differentiated from intermediate monocytes, which monitor the endothelial-blood interface and display a crawling behavior. Assumedly, both intermediate and nonclassical monocytes can invade the endothelium, renewing the macrophages and dendritic cell pool in each tissue, including arterial wall [6]. Of these, intermediate monocytes are characterized as the most proinflammatory because of the active production of the reactive oxygen species and inflammatory cytokines such as tumor necrosis factor (TNF)- $\alpha$ and interleukin (IL)-1 $\beta$. Furthermore, they demonstrate upregulated chemokine receptors relevant to atherosclerosis and high capacity of oxidized lowdensity lipoprotein-cholesterol (LDL-C) uptake.

In comparison to the apparent association between intermediate $\mathrm{CD} 14^{++} \mathrm{CD} 16^{+}$monocytes and cardiovascular events due to atherosclerosis [9-11], its relationship to metabolic disorders seems weak. Some studies have highlighted the positive correlation between 

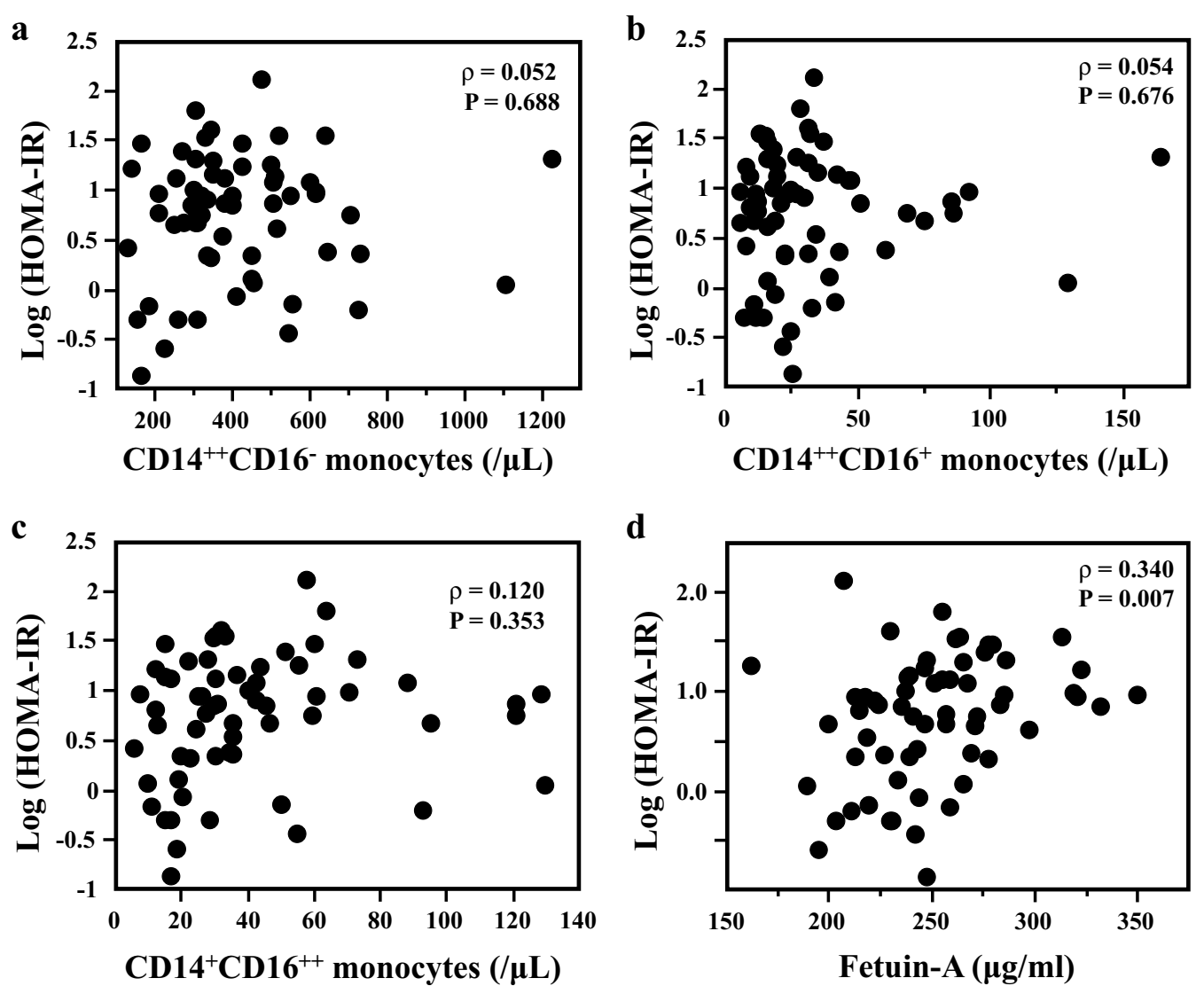

Fig. 2 The association of $\log (\mathrm{HOMA}-\mathrm{IR})$ with monocyte subsets $(\mathbf{a}-\mathbf{c})$ and serum levels of fetuin-A (d)

Table 2 Multivariate regression analyses of clinical factors possibly affecting the insulin resistance (HOMA-IR)

\begin{tabular}{|c|c|c|}
\hline & $\beta$ & $P$ \\
\hline Age & -0.005 & 0.975 \\
\hline Sex (Male) & 0.150 & 0.275 \\
\hline BMI & 0.305 & 0.034 \\
\hline $\mathrm{CD}_{14}^{++}{ }^{+} \mathrm{CD} 16^{-}$monocytes $(/ \mu \mathrm{L})$ & 0.015 & 0.939 \\
\hline $\mathrm{CD}_{14}^{++}{ }^{+} \mathrm{CD} 16^{+}$monocytes $(/ \mu \mathrm{L})$ & 0.037 & 0.871 \\
\hline $\mathrm{CD} 4^{+} \mathrm{CD} 16^{++}$monocytes $(/ \mu \mathrm{L})$ & 0.145 & 0.428 \\
\hline Fetuin-A (per 1-SD increase) & 0.313 & 0.016 \\
\hline$R^{2}$ & 0.219 & 0.052 \\
\hline
\end{tabular}

SD standard deviation

$\mathrm{CD}_{16}{ }^{+}$monocytes and obesity, a representative inflammatory state. Rogacev et al. demonstrated a significant correlation between higher $\mathrm{CD} 16^{+}$monocyte counts and higher BMI in healthy subjects [17]. Furthermore, a similar relationship between $\mathrm{CD}_{1} 6^{+}$monocytes and BMI was observed in obese and obese diabetic patients [18]. Remarkably, weight loss induced by diet intervention or gastric surgery led to a significant reduction in $\mathrm{CD}^{+} 6^{+}$monocytes [18]. Intriguingly, nonclassical $\mathrm{CD} 14^{+} \mathrm{CD} 16^{++}$monocytes correlated with $\mathrm{BMI}$ rather than intermediate $\mathrm{CD} 14^{++} \mathrm{CD} 16^{+}$monocytes.

Although we anticipated a positive correlation between proinflammatory intermediate monocytes and fetuin-A and insulin resistance, no association was determined in this study. Perhaps, the crucial issue might be whether proinflammatory intermediate monocytes in circulation could correspond to M1 macrophages in inflamed tissues [8]. To date, no apparent data illustrate one-to-one correspondence of monocyte subsets to macrophage polarity, M1 or M2. Recently, Jialal et al. reported the lack of association between plasma fetuin-A and the circulating monocyte TLR4 activity in humans [19]. Hence, it could be proposed that the inflammatory shift of monocyte subsets in circulation might not always be parallel to the proinflammatory polarization of M1 to M2 macrophages in adipose tissue [20]. The reason behind why intermediate monocytes cannot link to adipose tissues in inflamed condition is unclear. Perhaps, one primitive explanation for this condition could be the distance from the arterial wall. Since intermediate monocytes can directly penetrate the arterial wall, they can easily participate in 
plaque formation through their differentiation into macrophages [6]. Conversely, circulating monocytes might be modulated by various factors, including cytokines, in the lead up to reach adipose tissue, resulting in the discrepancy of proinflammatory characteristics between monocytes and macrophages.

Despite lacking any association between monocyte subsets and insulin resistance, fetuin- $\mathrm{A}$ is a negative contributor to the insulin sensitivity. Except for adipose tissue, fetuin-A has the potential to target other insulinsensitive tissues and can directly inhibit the tyrosine kinase activity of the insulin receptor in the liver and skeletal muscle $[21,22]$. Perhaps, this could illustrate the positive correlation between fetuin-A and insulin resistance independently of monocyte subsets and BMI.

There are several limitations to this study. First, the number of subjects was small, although we classified monocyte subsets using flow cytometry. Second, this was a cross-sectional study. Moreover, we mainly targeted obese patients with type 2 diabetes. The lack of lean subjects without diabetes disturbed further analyses. It might hide true relationship between monocyte subsets and inflammatory state. In addition, there was no information about characteristics of macrophages in adipose tissue. Therefore, additional studies are needed to compare circulating monocyte subsets with infiltrated macrophages in adipose tissue evaluated by biopsy.

\section{Conclusions}

To conclude, this study determined no correlation between monocyte subsets, including proinflammatory intermediate monocytes, and fetuin-A. Neither of the monocyte subsets demonstrated correlation with insulin resistance; however, fetuin-A showed a negative association with insulin sensitivity. Overall, this study suggests that monocyte subset counts in circulation could not parallel adipose tissue inflammation.

\begin{abstract}
Abbreviations
T2D: type 2 diabetes; AHSG: a2-Heremans Schmid glycoprotein; HOMA-IR: homeostasis model assessment for insulin resistance; FFA: free fatty acids; TLR4: Toll-like receptor-4; FPG: fasting plasma glucose; FIRI: fasting immunoreactive insulin; BMI: body mass index; SBP: systolic blood pressure; $\mathrm{Cr}$ : creatinine; LDL-C: low-density lipoprotein-cholesterol; TG: triglyceride; HDL-C: high-density lipoprotein-cholesterol; WBC: white blood cells; TNF: tumor necrosis factor; IL: interleukin.
\end{abstract}

\section{Authors' contributions}

$\mathrm{SM}, \mathrm{KM}, \mathrm{KM}, \mathrm{MN}$, and RK contributed to the data collection, statistical analysis, and drafting the manuscript. YY, YK, TM, TS, ME, and MI participated in conducting the study, interpretation of data, and drafting the manuscript. KMor reviewed/edited the manuscript. All authors read and approved the final manscript.

\section{Author details}

${ }^{1}$ Department of Metabolism, Endocrinology and Molecular Medicine, Osaka City University Graduate School of Medicine, Osaka, Japan. ${ }^{2}$ Department of Nephrology, Osaka City University Graduate School of Medicine, 1-4-3, Asahi-machi, Abeno-ku, Osaka 545-8585, Japan. ${ }^{3}$ Department of Vascular Medicine, Osaka City University Graduate School of Medicine, Osaka, Japan.

\section{Competing interests}

The authors declare that they have no competing interests.

\section{Availability of data and materials}

K. Motoyama is the guarantor of this work; he has full access to all data in this study and takes responsibility for data.

\section{Consent for publication}

Not applicable.

\section{Ethics approval and consent to participate}

This study was approved by the Ethics Committee of Osaka City University Hospital. We obtained informed consent from all patients in this study before participation.

\section{Funding}

This study received no specific funding.

\section{Publisher's Note}

Springer Nature remains neutral with regard to jurisdictional claims in published maps and institutional affiliations.

Received: 20 December 2017 Accepted: 10 February 2018

Published online: 27 March 2018

\section{References}

1. Mori K, Emoto M, Inaba M (2011) Fetuin-A: a multifunctional protein. Recent Pat Endocr Metab Immune Drug Discov 5(2):124-146

2. Stefan N, Haring HU (2013) The role of hepatokines in metabolism. Nat Rev Endocrinol 9(3):144-152

3. Mori K, Emoto M, Inaba M (2012) Fetuin-A and the cardiovascular system. Adv Clin Chem 56:175-195

4. Pal D, Dasgupta S, Kundu R, Maitra S, Das G, Mukhopadhyay S et al (2012) Fetuin-A acts as an endogenous ligand of TLR4 to promote lipid-induced insulin resistance. Nat Med 18(8):1279-1285

5. Chatterjee P, Seal S, Mukherjee S, Kundu R, Mukherjee S, Ray S et al (2013) Adipocyte fetuin-A contributes to macrophage migration into adipose tissue and polarization of macrophages. J Biol Chem 288(39):28324-28330

6. Heine GH, Ortiz A, Massy ZA, Lindholm B, Wiecek A, Martinez-Castelao A et al (2012) Monocyte subpopulations and cardiovascular risk in chronic kidney disease. Nat Rev Nephrol 8(6):362-369

7. Ziegler-Heitbrock L, Ancuta P, Crowe S, Dalod M, Grau V, Hart DN et al (2010) Nomenclature of monocytes and dendritic cells in blood. Blood 116(16):e74-e80

8. Yang J, Zhang L, Yu C, Yang XF, Wang H (2014) Monocyte and macrophage differentiation: circulation inflammatory monocyte as biomarker for inflammatory diseases. Biomark Res 2(1):1

9. Heine GH, Ulrich C, Seibert E, Seiler S, Marell J, Reichart B et al (2008) CD14(++)CD16+ monocytes but not total monocyte numbers predict cardiovascular events in dialysis patients. Kidney Int 73(5):622-629

10. Rogacev KS, Seiler S, Zawada AM, Reichart B, Herath E, Roth D et al (2011) CD14++CD16+ monocytes and cardiovascular outcome in patients with chronic kidney disease. Eur Heart J 32(1):84-92

11. Rogacev KS, Cremers B, Zawada AM, Seiler S, Binder N, Ege P et al (2012) CD14++CD16+ monocytes independently predict cardiovascular events: a cohort study of 951 patients referred for elective coronary angiography. J Am Coll Cardiol 60(16):1512-1520

12. American Diabetes A (2004) Diagnosis and classification of diabetes mellitus. Diabetes Care 27(Suppl 1):S5-S10

13. Imai E, Horio M, Nitta K, Yamagata K, Iseki K, Tsukamoto Y et al (2007) Modification of the modification of diet in renal disease (MDRD) study equation for Japan. Am J Kidney Dis 50(6):927-937 
14. Mori K, Emoto M, Yokoyama H, Araki T, Teramura M, Koyama H et al (2006) Association of serum fetuin-A with insulin resistance in type 2 diabetic and nondiabetic subjects. Diabetes Care 29(2):468

15. Mori K, Emoto M, Araki T, Yokoyama H, Lee E, Teramura M et al (2008) Effects of pioglitazone on serum fetuin-A levels in patients with type 2 diabetes mellitus. Metabolism 57(9):1248-1252

16. Matthews DR, Hosker JP, Rudenski AS, Naylor BA, Treacher DF, Turner RC (1985) Homeostasis model assessment: insulin resistance and beta-cell function from fasting plasma glucose and insulin concentrations in man. Diabetologia 28(7):412-419

17. Rogacev KS, Ulrich C, Blomer L, Hornof F, Oster K, Ziegelin M et al (2010) Monocyte heterogeneity in obesity and subclinical atherosclerosis. Eur Heart J 31(3):369-376

18. Poitou C, Dalmas E, Renovato M, Benhamo V, Hajduch F, Abdennour M et al (2011) CD14dimCD16+ and CD14+CD16+ monocytes in obesity and during weight loss: relationships with fat mass and subclinical atherosclerosis. Arterioscler Thromb Vasc Biol 31(10):2322-2330

19. Jialal I, Devaraj S, Adams-Huet B (2016) Plasma fetuin-A does not correlate with monocyte TLR4 in humans. Diabetologia 59(1):222-223

20. Mukhopadhyay S, Bhattacharya S (2016) Plasma fetuin-A triggers inflammatory changes in macrophages and adipocytes by acting as an adaptor protein between NEFA and TLR-4. Diabetologia 59(4):859-860

21. Mathews ST, Chellam N, Srinivas PR, Cintron VJ, Leon MA, Goustin AS et al (2000) Alpha2-HSG, a specific inhibitor of insulin receptor autophosphorylation, interacts with the insulin receptor. Mol Cell Endocrinol 164(1-2):87-98

22. Mathews ST, Singh GP, Ranalletta M, Cintron VJ, Qiang X, Goustin AS et al (2002) Improved insulin sensitivity and resistance to weight gain in mice null for the Ahsg gene. Diabetes 51(8):2450-2458

\section{Submit your manuscript to a SpringerOpen ${ }^{\circ}$ journal and benefit from:}

- Convenient online submission

- Rigorous peer review

- Open access: articles freely available online

- High visibility within the field

- Retaining the copyright to your article

Submit your next manuscript at $\boldsymbol{\nabla}$ springeropen.com 